\title{
Applications of Clonal Selection Algorithm Based on Tabu Criteria in Combinatorial Optimization
}

\author{
Yongfei Miao, ${ }^{1, a}$, Yufu Yin ${ }^{2, b}$ and Yunpeng Wang ${ }^{3, c}$ \\ ${ }^{1}$ Wuhan University of Technology, Wuhan 430070, Hubei, China \\ ${ }^{2}$ Air Force Engineering University, Xi'an 710051, Shanxi, China \\ ${ }^{3}$ Dalian Air Force Sergeant School of Communication, Dalian 116600, Liaoning, China \\ ayongfei1900@163.com, byf_yin2008@163.com, ‘yp_wang2000@163.com
}

Keywords:Tabu Algorithm; Clonal Selection Algorithm; Combinatorial Optimization; Hypermutation

\begin{abstract}
Clonal selection algorithm has a shortcoming when solving the problem of combinatorial optimization, which is that the antibody diversity of population evolution declines in later stage. Therefore, this paper has improved the selection operator of this algorithm and introduced tabu criteria. What's more, the benchmark of a typical combinatorial optimization problem is combined to carry out simulation analysis on this algorithm. The result shows that the improved clonal selection algorithm owns a better global convergence, stability and quick convergence ability to solve combinatorial optimization problems.
\end{abstract}

\section{Introduction}

Combinatorial optimization concerns about finding out the best arrangement, grouping, sequence and selection of discrete time through the study of mathematical methods. It is a classic and important branch of operations research. And the issues it studies relate to many areas such as information technology, macro-economic management, industrial engineering, transportation, military technology, communication networks, etc. Lots of optimization questions in real life is to choose the best one from a limited number of conditions and so that most actual optimization problems can be classified as combinatorial optimization problems, for example, knapsack problem $^{[1]}$, traveling salesman problem(TSP $)^{[2]}$, job scheduling problem(JSP $)^{[3-5]}$, robot path planning $^{[6]}$, pattern recognition ${ }^{[7]}$ and assembly sequence planning ${ }^{[8]}$ and so on.

Over recent years, lots of papers have focused on Artificial Immune Systems (AIS), which have covered reviewing AIS in general, or much more specific aspects of AIS such as data mining , network security, the application of AIS, theoretical aspects and modeling in AIS. AIS have become known as an area of computer science and engineering which uses immune system metaphors for the creation of novel solutions to problems.

Within AIS, there are common and well understood algorithms based on specific aspects of immunology. Clonal selection algorithms are the most widely employed algorithms, which take their inspiration form how cells of the adaptive immune system (specifically, cells called B-cells) interact with the "antigen"(something that causes an immune response) and then undergo a process of cloning, mutation and selection. In this paper, a clonal selection algorithm based on tabu criteria (CSAbT) is presented, which aims to solve traveling salesman problem. This algorithm has improved the selection operator and adds in tabu search algorithm. The experiment results show that artificial immune algorithm has been greatly improved in the aspects of keeping the diversity of population and quickly converging to the optimal solution.

Section 2 mainly describes the proposed algorithm of solving TSP. Then experiments and experimental comparison results are presented in Section 3. Finally, section 4 makes a simple conclusion. 


\section{CSAbT in solving TSP problems}

Traveling salesman problem, the most representative classic problem of combinatorial optimization, can be described as follows: to calculate the shortest path of going through all the cities on the basis of the given cities and the distances between every two cities. In graph theory, TSP can be described as follows: a graph is given, $G=(V, A)$ and $V$ is the vertex set and $A$ represents the side set, which is composed of the sides between every two vertices. The distances between every two vertices are given and the shortest circuit is required to be calculated. The one means the shortest circuit when every vertex is passed only once.

\subsection{Antibody coding, calculation of the fitness and the affinity}

Antibody coding adopts the form of float encoding method, which means forming coded string with city numbers. The fitness function of the antibody - antigen is defined as follows:

$$
\begin{aligned}
& \operatorname{Dis}_{i}=\sum_{i=1}^{N-1} d\left(v_{i}, v_{i+1}\right)+d\left(v_{i}, v_{N}\right) \\
& \text { Fit }_{i}=76.5 L \sqrt{N} / \text { Dis }_{i}
\end{aligned}
$$

Fit $t_{i}, \quad$ Dis $i$ and $d\left(v_{i}, v_{i+1}\right)$ represent sufficiency, path length and the distance between city $i$ and city $i+1$. Then $L$ means the side-length of the minimum square to surround all the cities and $N$ is the number of the cities.

The Euclidean method is selected to measure the affinities among antibodies. The Euclidean distance between one individual $P_{1}\left(m_{1}, m_{2}, \ldots, m_{N}\right)$ and another individual $P_{2}\left(n_{1}, n_{2}, \ldots, n_{N}\right)$ can be calculated by this expression:

$$
\operatorname{Dis}_{E}\left(P_{1}, P_{2}\right)=\sqrt{\sum_{i=1}^{N}\left(m_{i}-n_{i}\right)}
$$

It reflects that the smaller the Euclidean distance, the higher the individual affinity. And the Euclidean distance goes in zero when two individuals are just the same.

\subsection{Immune selection operation}

Set the measuring vector as $K_{k y}$ while the fitness between the antibody and the antigen is normalized. Then we can see from $\mathrm{Eq}(4)$ that the higher the fitness between the antibody and the antigen, then the shorter the distance when the cities are passed by and the higher corresponding normalization metric parameters.

Set the measuring vector as $K_{k t}$, Then we can see from Eq(5) that the lower the affinity between the antibodies, the lower the degree of similarity between the antibodies and the farther corresponding Euclidean distance and the higher normalized parameter. Then selective probability $P_{s}$ is defined as follows:

$$
\begin{aligned}
K_{k y} & =\frac{D i s_{i}}{\sum_{i=1}^{N} D i s_{i}} \quad(i=1,2, \ldots, \text { Popsize }) \\
K_{k t} & =\frac{\sum_{j=1}^{N} D i s_{E}\left(P_{i}, P_{j}\right)}{\sum_{i=1}^{N} \sum_{j=1}^{N} D i s_{E}\left(P_{i}, P_{j}\right)} \quad(i, j=1,2, \ldots, \text { Popsize }) \\
P_{s} & =\lambda K_{k y}+(1-\lambda) K_{k t}
\end{aligned}
$$

$\lambda$ represents the selection ratio parameter of the fitness and the affinity. In the early period of algorithm iteration, the diversity of antibody is great and so that more excellent individuals are keen to be kept. In the later period of algorithm iteration, the differences among antibodies have lost and individuals with lower affinities are keen to be kept. Therefore, $\lambda$ makes dynamic adjustment with the iteration and it will decrease gradually when the number of iterations increases. 


\subsection{Crossover, hypermutation and tabu operation}

Crossover operator adopts Edge Recombination Crossover. Compared with other interleaved modes, it can make the next generation inherit good qualities from the parent much better.

Hyper-mutation operator is to clone the antibody cell at corresponding number according to the degree of the affinity between the antibody and the antigen.

$$
N_{i}=\operatorname{int}\left(N_{c} \cdot \frac{f\left(X_{i}\right)}{\sum_{j=1}^{N} f\left(X_{j}\right)}\right)
$$

Here $N_{c}$ is the scale of the total antibody population after cloning, then int(•) means integral function and $f\left(X_{i}\right)$ stands for the affinity value of the $i$ individual. $N_{i}$ is the cloning number of the $i$ individual. Therefore it can be concluded that after the hypermutation, individuals with high affinities have generated more mirror images, preserved more good characteristics and enhanced the searching ability of solution space.

$C S A b T$ has simulated the memory mechanism of good individuals and stored individuals with high affinities in the memory set. In the process of population iteration, the memory set will be updated if better individuals appear and the memory set will remain unchanged if the individual affinities of the offspring are lower than those of the memory set. On the one hand, the direction of convergence algorithm is ensured and the population will be on a global minimum of evolution. On the other hand, it will easily cause individual diversity reduction and serious population degradation. Especially in the later algorithm evolutionary, individual affinities of the population are similar or identical with those of the memory set, which make the algorithm do some circuitous search around local optimum points and so as to reduce the efficiency of algorithm convergence. Therefore, this algorithm has introduced tabu criteria, whose main idea is to mark obtained local optimum solution and to avoid from such local optimum solution during further iterative process. Add those individuals whose affinities won't increase during recent iterative process into tabu list. Individuals will not be added in the population if they are randomly generated in the neighborhood of the area where individuals in tabu list are generated. The individuals in tabu list will be amnestied if their tabu numbers exceed a certain threshold. Aspiration criteria aims to further expand search space and make good individuals participate in immune process again and ensure persistent search ability against the original space. So individuals which are added randomly have better spatial distribution and circuitous search can be reduced. Then more local optimum points can to be searched, the global search ability of the algorithm gets strengthened and the convergence speed is accelerated.

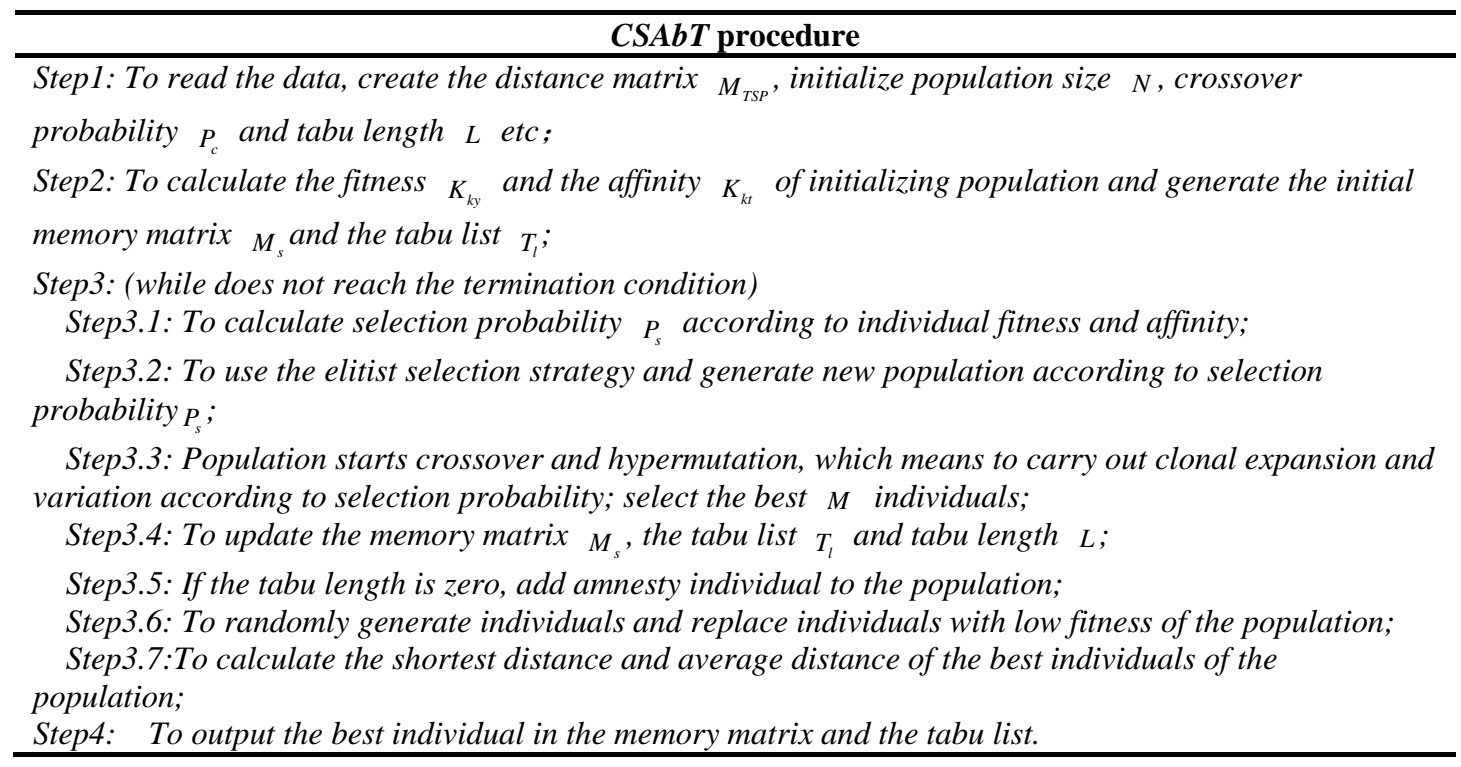


Figure 1. Procedure of CSA $b \mathrm{~T}$ algorithm

\section{Experiments and Results}

In order to test the performance of CSAbT algorithm, some experiments are conduced on six TSP problems named Ulysses16, Bays29, Att48, Eil51, Pr120 and Gr202, which come from international TSPLib benchmarks or from papers ${ }^{[9-11]}$.

We use CSAbT to solve all the test TSP problems which are given above. CSAbT adopts an elitist selection operation as the selection operator, and adopts ordered crossover operator. The maximum iterations are set to be 2000 and all experiments run 20 times. Parameters are set as follows:

Parameters of CSAbT:

- Population size: $N=100$; Memory set size: $C M=20$; Tabu length: $L=5$;

- Crossover factor: $P_{c}=0.4$; Mutation factor: $P_{m}=0.03$;

Table 1 Experiment Results of CSAbT

\begin{tabular}{cccc}
\hline & \multirow{2}{*}{$\begin{array}{c}\text { Optimal } \\
\text { value } \\
\text { Reported }\end{array}$} & Optimum & Worst \\
\cline { 3 - 4 } & 74.1087 & 73.9876 & 73.9998 \\
Ulysses16 & 9074.148 & 9074.148 & 9197.0465 \\
Bays29 & 33523.7085 & 33784.027 \\
Att48 & 423.7085 & 429.5303 & 436.3626 \\
Eil51 & 1666.5 & 1666.5 & 1694.8733 \\
Pr120 & 549.99 & 503.1994 & 515.4646 \\
Gr202
\end{tabular}

Table 2 The shortest path obtained by CSAbT

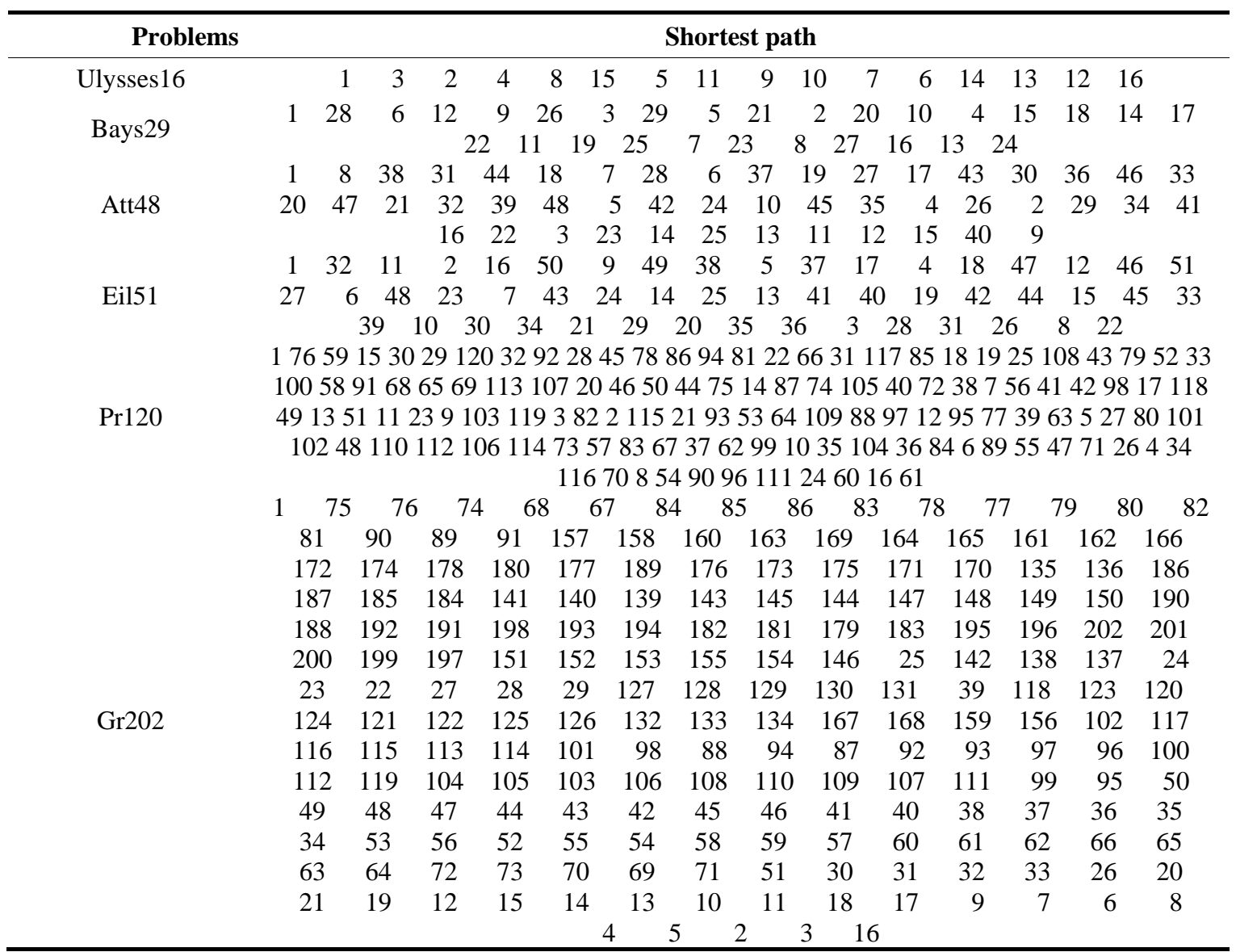


Take 51cities and 202 cities for example, their shortest path obtained by CSAbT is shown as follows:
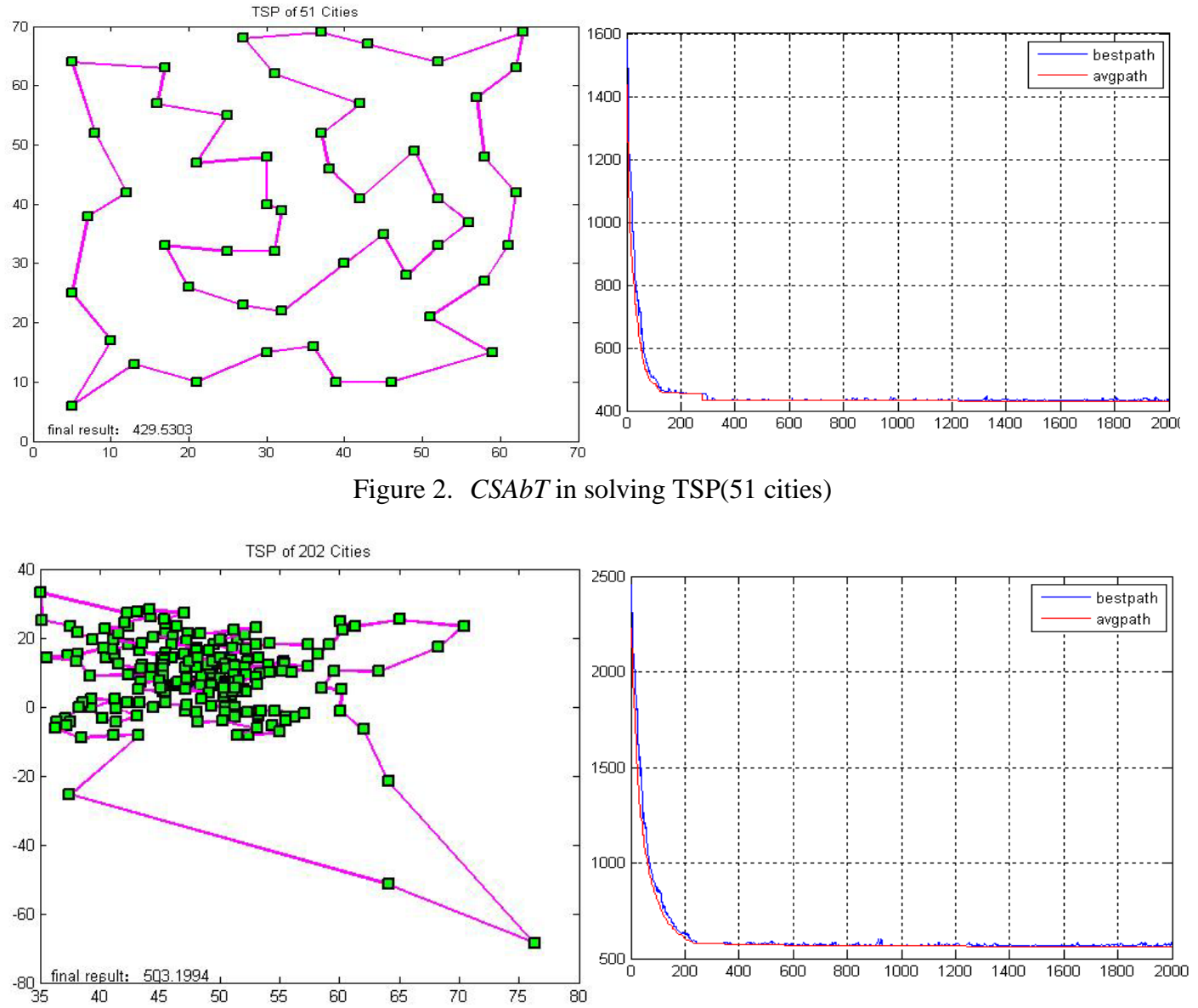

Figure 3. $C S A b T$ in solving TSP(202 cities)

The computational results obtained by the CSAbT algorithm are reported in Table1-2. From the results we can see that $C S A b T$ can quickly converge to the approximate solution and finally reach the global optimum. In most cases, CSAbT can find a better path than the optimal path which has been reported. In addition, the rate of convergence has been improved greatly. This shows that $C S A b T$ is an effective algorithm to solve TSP.

\section{Conclusions}

This paper has put forward a clonal selection algorithm based on tabu criteria, which makes full use of the advantages of tabu algorithm and clonal selection algorithm. It has corrected some shortcomings of clonal selection algorithm, which are caused by circuitous search. For instance, the searching efficiency gets lower and it's easy to fall into the local optimal solution. The diversity of the population decreases in the later searching period and the degradation of population gets serious, etc. CSAbT has improved the convergence speed of the algorithm and better reflects these mechanisms such as parallel, self-organizing and immune memory of artificial immune system. The simulations demonstrate that this algorithm has well kept the diversity of the population, avoids trapping in local optimum problem and finally has reached the objectives of global optimization and rapid convergence. In a word, it shows an excellent performance.

\section{References}


[1] F. Zhao,Y. Ma and J. Zhang. "Solving 0-1 Knapsack Problem Based on Immune Clonal Algorithm and Ant Colony Algorithm”, 2012 International Conference on Communication, Electronics and Automation Engineering Advances in Intelligent Systems and Computing Volume 181, 2013, pp. 1047-1053.

[2] R. Wang, X. Zhou, L. Zhao and Z. Xia. "An Ant System with Two Colonies and Its Application to Traveling Salesman Problem”. IEEJ Transactions on Electronics, Information and Systems. Vol. 132 No.12, 2012, pp. 2043-2050.

[3] A. Ponsich, A. Carlos and C. Coello. "A hybrid Differential Evolution-Tabu Search algorithm for the solution of Job-Shop Scheduling Problems”. Applied Soft Computing. Volume 13, Issue 1, January 2013, pp. 462-474.

[4] X. Zuo, C. Wang and W. Tan. "Two heads are better than one: an AIS- and TS-based hybrid strategy for job shop scheduling problems”.Int J Adv Manuf Technol(2012)63,pp.155 168.

[5] R. G. Babukarthik, R. Raju and P. Dhavachelvan. "Hybrid Algorithm for Job Scheduling: Combining the Benefits of ACO and Cuckoo Search”. Intelligent Systems and Computing Volume 177, 2013, pp. 479-490 .

[6] M. Yuan, S. Wang and C. Wu. "A Novel Immune Network Strategy for Robot Path Planning in Complicated Environments”. J Intell Robot Syst 60, 2010, pp. 111-131.

[7] F. Castiglione, S. Motta and G. Nicosia. "Pattern Recognition by Primary and Secondary Respone of an Artificial Immune System”. Theory Biosci 120, 2001, pp. 93-106.

[8] C. Chang, H. Tseng and L. Meng. “Artificial immune systems for assembly sequence planning exploration”. Engineering Applications of Artificial Intelligence 22, 2009, pp. 1218-1232.

[9] A. Levin and U. Yovel. "Local search algorithms for multiple-depot vehicle routing and for multiple travelling salesman problems with proved performance guarantees”. J Comb Optim, Dec. 2012.

[10]H. Zhao. “Application of artificial immune algorithm in TSP”, Southwest Jiao Tong University Master Degree Thesis,2010.

[11]Z. Liu. "Immune clone selection algorithm research and its application”,Hunan University Master Degree Thesis,2010. 